\title{
Development of a yeast heterologous expression cassette based on the promoter and terminator elements of the Eremothecium cymbalariae translational elongation factor 1a (EcTEF1) gene
}

\author{
Tomas Linder $^{1}$ (D) \\ Received: 8 October 2017 / Accepted: 22 March 2018 / Published online: 29 March 2018 \\ (c) The Author(s) 2018
}

\begin{abstract}
A new expression cassette (ECO) consisting of the fused $5^{\prime}$ and $3^{\prime}$ intergenic regions (IGRs) of the Eremothecium cymbalariae translational elongation factor $1 \alpha(E c T E F 1)$ gene was evaluated through expression of the bacterial hygromycin B phosphotransferase $(h p h)$ resistance gene in the common baker's yeast Saccharomyces cerevisiae. Progressively shorter versions of the $h p h$-containing EC cassette ( $h p E E C 1$ though $h p h E C 6$ ) with trimmed 5' and 3' EcTEF1 IGRs were tested for their ability to confer resistance to hygromycin B in $S$. cerevisiae. Hygromycin B resistance was retained in all six generated $h p h E C$ variants up to a concentration of $400 \mathrm{mg} / \mathrm{L}$. The $h p h E C 6$ cassette was the shortest cassette to be assayed in this study with 366 and 155 bp of the EcTEF 1 5' and 3' IGRs, respectively. When tested for deletion of the S. cerevisiae proline oxidase gene PUTI, the $h p h E C 6$ cassette was shown to successfully act as a selection marker on hygromycin B-containing medium. The hphEC6 cassette could be placed immediately adjacent to a kanMX4 G418 disulfate resistance marker without any discernable effect on the ability of the yeast to grow in the presence of both hygromycin B and G418 disulfate. Co-cultivation experiments under non-selective conditions demonstrated that a PUTl deletion strain carrying the $h p h E C 6$ cassette displayed equivalent fitness to an otherwise isogenic PUT1 deletion strain carrying the kanMX4 cassette.
\end{abstract}

Keywords Heterologous expression $\cdot$ Hygromycin $\cdot$ Selection marker $\cdot$ Yeast

$\begin{array}{ll}\text { Abbreviations } \\ \text { EcTEF1 } & \begin{array}{l}\text { Eremothecium cymbalariae translational factor } \\ 1 \alpha \text { gene }\end{array} \\ & \begin{array}{l}\text { Hygromycin phosphotransferase gene } \\ h p h\end{array} \\ \text { IGR } & \text { Intergenic region } \\ \text { ORF } & \text { Open reading frame } \\ \text { RGE } & \text { Rapid growth element } \\ \text { RP } & \text { Ribosomal protein } \\ \text { RRPE } & \text { Ribosomal RNA processing element } \\ \text { ScPUT1 } & \text { Saccharomyces cerevisiae proline oxidase gene }\end{array}$

Electronic supplementary material The online version of this article (https://doi.org/10.1007/s13205-018-1224-0) contains supplementary material, which is available to authorized users.

Tomas Linder

tomas.linder@slu.se

1 Department of Molecular Sciences, Swedish University of Agricultural Sciences, Box 7015, 75007 Uppsala, Sweden

\section{Introduction}

During the past 4 decades, the genetic engineering of yeasts and other microorganisms has become a multi-billion dollar industry. The common baker's yeast Saccharomyces cerevisiae is the most commonly used yeast systems in synthetic biology applications although other species of budding yeasts (phylum Ascomycota, sub-phylum Saccharomycotina) are gaining in popularity. The traditional approaches to genetic engineering in yeast require condition-specific selection marker genes for the isolation of successful transformants. The design of dominant selection markers such as antibiotic resistance genes tends to employ promoter sequences from constitutively active and highly transcribed "house-keeping genes" such as those involved in protein synthesis, central carbon metabolism, or cell cycle progression. Strong constitutive promoters may also be desirable for some synthetic biology applications such as the rewiring of metabolic pathways or increasing pathway flux.

Translational elongation factor $1 \alpha$ (Tef1) is one of the most abundant proteins in actively dividing yeast cells (Ghaemmaghami et al. 2003) and the strong activity of 
the TEF 1 gene promoter has lead to the development of a number of expression cassettes based on the TEF1 intergenic regions (IGRs) from yeasts (Müller et al. 1998; Ahn et al. 2007). The 5' and 3' IGRs of the TEF1 gene from the filamentous yeast Eremothecium gossypii (syn. Ashbya gossypii) has become one of the most widely used expression cassettes (commonly designated " $M X$ ") for strong, constitutive expression of dominant selection markers in the genetic engineering of yeasts (Steiner and Philippsen 1994; Wach et al. 1994). The $M X$ cassette has been shown to work for the expression of resistance genes in a wide spectrum of yeast species including the fission yeast Schizosaccharomyces pombe (Bähler et al. 1998) despite its very distant evolutionary relationship to budding yeasts (Beimforde et al. 2014).

However, the ubiquity of the $M X$ expression cassette in the design of dominant selection markers for the genetic engineering of yeasts often results in transgenic strains that are modified several times with multiple $M X$ cassettes carrying different resistance genes. The repeated use of the $M X$ cassette or any other sequence element within genomic integration constructs will progressively decrease targeting efficiency of subsequent transformations within the same yeast genome (Davidson and Schiestl 2000). Accumulation of integrated sequence elements that contain longer stretches of identical sequence also introduces a risk of undesirable chromosomal rearrangements during genetic crosses and may introduce artifacts in high-throughput genetic analyses such as synthetic genetic arrays. Repeated use of a single or a limited sub-set of expression cassettes can also be problematic in synthetic biology applications such as heterologous expression of multigene biosynthetic pathways, where tandem arrays containing identical regulatory sequences can result in recombination-mediated excision of transgenes.

In the case of selection markers, one approach to circumvent the accumulation of identical regulatory elements within the genome of a yeast strain has been to remove the selection marker post-integration through heterologous expression of a recombinase such as Cre (Güldener et al. 1996). This approach has the added advantage of enabling repeated use of the same selection marker and thereby selection strategy. However, repeated recombinase-mediated selection marker recycling in $S$. cerevisiae has been shown to cause significant chromosomal rearrangements and gene loss (Solis-Escalante et al. 2015). An alternative approach is to introduce expression cassettes containing new promoter and terminator elements. For instance, there have been variants of the $M X$ cassette described where the EgTEF1 terminator sequence have been substituted for that of other genes such as the $S$. cerevisiae $A D H 1$ and $C Y C 1$ genes (Janke et al. 2004). Although this reduces the risk of unwanted recombination events with any pre-existing $M X$ cassettes within the yeast genome, it also introduces a new risk of recombination events with the endogenous terminator elements of the
$A D H 1$ and $C Y C 1$ genes if these cassettes were to be used in the transformation of $S$. cerevisiae.

Newly sequenced yeast genomes are valuable resource for new regulatory sequences for heterologous expression of selection markers and other transgenes. As IGRs are noncoding, they evolve faster than coding gene sequences while retaining their regulatory function in related species. Thus, a promoter or terminator element can be transplanted into the genome of a more distantly related species and function, even though the overall sequence homology between promoters may be beyond detection. The present study sought to develop a new constitutive expression cassette based on the IGRs of the TEF 1 gene from the filamentous yeast Eremothecium cymbalariae, which is the closest relative of $E$. gossypii with a sequenced genome at the time of writing (Wendland and Walther 2011).

\section{Methods}

\section{Yeast integration constructs}

PCR primers used in this study are listed in Table 1. An ScPUT1 targeting sequence ("Sc_Aputl") consisting of two fused 200-bp segments of the ScPUT1 5' and 3' IGRs (GenBank accession AEHG01000269, residues 13,597-13,796 and 15,710-15,909, respectively) was synthesized de novo by GenScript (NJ, USA) and inserted into EcoRI/HindIII-cut pUC57 (GenBank accession Y14837) to produce the plasmid pUC57-Sc_sputl (Fig. 1a). The $S c \_\Delta p u t 1$ sequence was amplified from pUC57-Sc_Aput1 using primers ScPUT1 3' fwd and $S c P U T 15^{\prime}$ rev. The $S c \_\Delta p u t 1$ amplification product was cut with BglII and SmaI and inserted in the reverse orientation into BglII/SmaI-cut pFA6a-kanMX4 (GenBank accession AJ002680; Wach et al. 1994) to produce the plasmid pFA6a-Sc_sput1-kanMX4 (Fig. 1b, c).

A fusion of the full-length $5^{\prime}$ and $3^{\prime}$ IGRs of EcTEF 1 gene (GenBank accession NC_016451, reverse complement of residues 841,757-842,469 and 840,021-840,379, respectively) was synthesized de novo by GenScript (NJ, USA) and inserted into EcoRI/HindIII-cut pUC57 to produce the plasmid pUC57-ECO (Fig. 1d). The ECO cassette also includes the first 12 basepairs of the EcTEF1 open reading frame (ORF) corresponding to the amino acid sequence MGKE. The $h p h$ coding sequence (GenBank accession K01193) was amplified from the plasmid pFA6a-hphNT1 (Janke et al. 2004) using primers $h p h$ fwd and $h p h$ rev. The $h p h$ amplification product was cut with Ecl136II and inserted into Ec1136II-cut pUC57-ECO to produce the plasmid pUC57-hphECO.

Six $h p h E C$ variants ( $h p h E C 1-h p h E C 6$, Fig. 1e) were generated by PCR amplification of pUC57-hphECO using all six possible pair-wise combinations of forward primers EcTEF 1 
Table 1 Primers used in this study
Fig. 1 Plasmid design. a Design of the S. cerevisiae PUT1 targeting cassette (Sc_sput1) as inserted into the EcoRI/ HindIII site of the pUC57 plasmid. b Sc_sputl targeting cassette was inserted into the pFA6a-kanMX4 plasmid in its reverse orientation. c Resulting pFA6a-Sc_Aput1-kanMX4 plasmid with insertion sites I and II highlighted in grey. Plasmid components are not drawn to scale. d Design of the $E$. cymbalariae TEF1 expression cassette $(E C O)$ as inserted into the EcoRI/HindIII site of the pUC57 plasmid. The first four codons of the EcTEF1 ORF are highlighted in grey. e Schematic representation of the six $h p h E C$ cassettes assayed in this study. The lengths (bp) of 5' and 3' IGRs are indicated and drawn to scale. The $h p h$ coding sequence is not drawn to scale

\begin{tabular}{|c|c|}
\hline Primer name & Sequence $\left(5^{\prime} \rightarrow 3^{\prime}\right)$ \\
\hline ScPUT1 3' fwd & GCG CGC AGA TCT ATT ATC AAC TCT TAT GCA CAA G \\
\hline ScPUT1 5' rev & GCG CGC CCG GGT GAT AAG GGA AAT AGC GCC AC \\
\hline ScPUT1 5' ctrl rev & TGT TCC GAT CAG CAT TAC ATG \\
\hline ScPUTl ctrl rev & GAT GCT GTG AGA TCT GAT AAT GG \\
\hline ScPUT1 3' ctrl fwd & ATT TCA TCA TCC TGA GTA GCA GTA \\
\hline pFA6 ctrl fwd & ACT GAG AGT GCA CCA TAT GGA \\
\hline$h p h$ fwd & GCG CGC GAG CTC AAA AAG CCT GAA CTC ACC GCG A \\
\hline$h p h \mathrm{rev}$ & GCG CGC GAG CTC ATT CCT TTG CCC TCG GAC GAG T \\
\hline EcTEF1 5' 693 fwd & GCG CGC AAT TGC CTA TCG CTG ATC CTC ATG TA \\
\hline EcTEF1 5' $581 \mathrm{fwd}$ & GCG CGC AAT TGA CAT GCC GCA CAC AGC ACA GA \\
\hline EcTEF1 5' 366 fwd & GCG CGC AAT TGT ATG CGC AGA TTG TGT ATC T \\
\hline EcTEF1 5' 366 fwd 2 & GCG CGC GGA TCC GTA TGC GCA GAT TGT GTA TCT \\
\hline EcTEF1 3' 339 rev & GCG CGC GAT ATC TAA TTG TAA ACT TCA TGA CT \\
\hline EcTEF1 3' $155 \mathrm{rev}$ & GCG CGC GAT ATC CTG AGA TAT GCG CTC CTA CT \\
\hline
\end{tabular}

a

pUC57-Sc_sput1

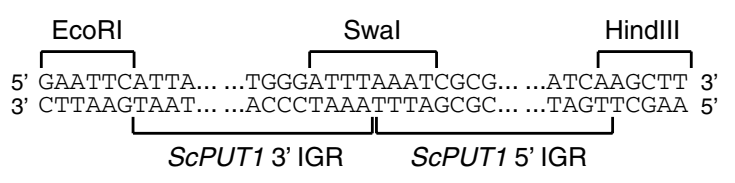

b

$\Delta$ put1

C
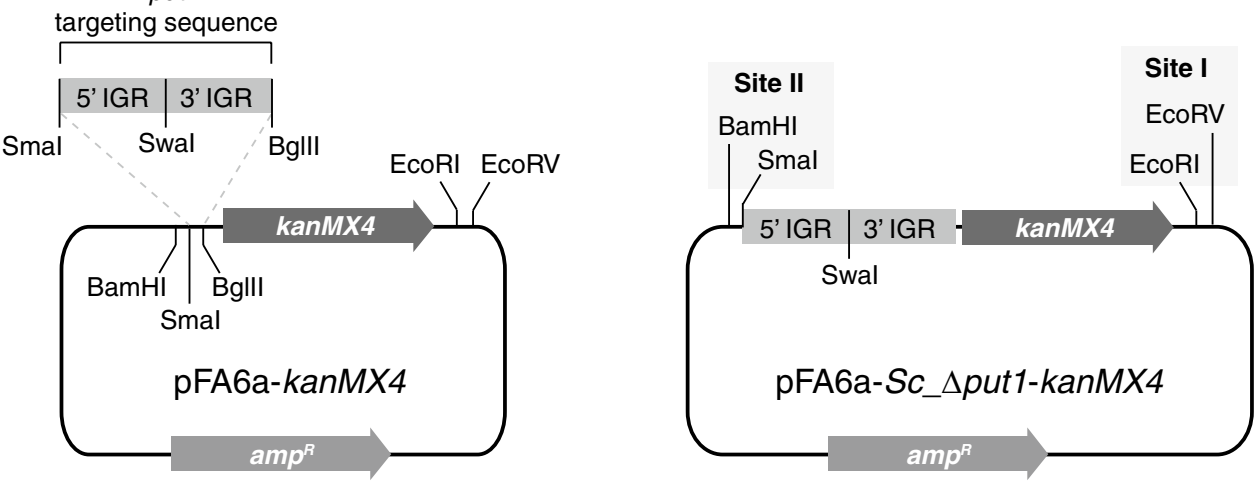

d

pUC57-ECO

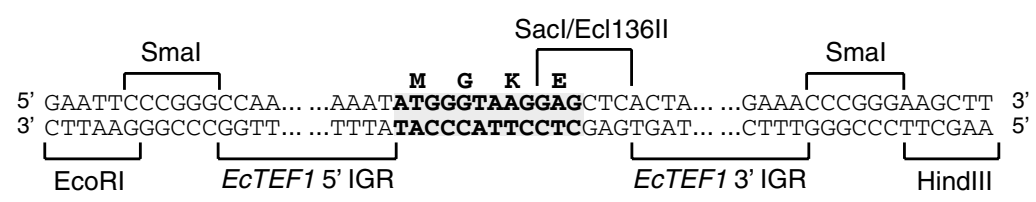

$\mathbf{e}$

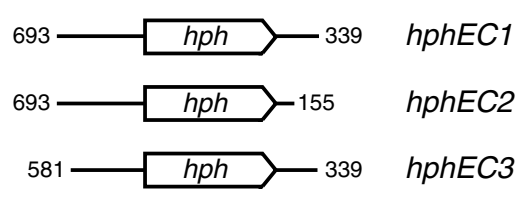

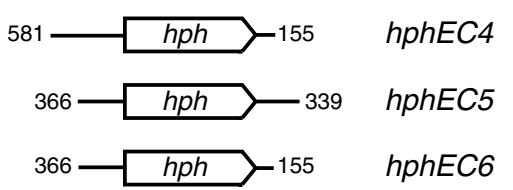


5' 693 fwd, EcTEF1 5' 581 fwd and EcTEF1 5' 366 fwd with reverse primers EcTEF1 3' 339 rev and EcTEF1 3' 155 rev. Each $h p h E C$ variant ( $h p h E C 1$ through $h p h E C 6$ ) was cut with MfeI and EcoRV and inserted into EcoRI/EcoRV-cut pFA6a-Sc_Aputl-kanMX4 (site I in Fig. 1c) to produce plasmids pFA6a-Sc_Aput1-kanMX4-hphEC1 through -hphEC6.

An "expression cassette-only" control plasmid (pFA6a$\left.S c \_\Delta p u t 1-k a n M X 4-E C 1\right)$ was generated by first amplifying the 5' and 3' EcTEF1 IGRs from the pUC57-ECO plasmid using primers EcTEF1 5' 693 fwd and EcTEF1 3' 339 rev. The resulting $E C l$ amplification product was then digested with MfeI and EcoRV and inserted into EcoRI/EcoRV-cut pFA6a-Sc_Aput1-kanMX4.

An integration plasmid where the kanMX4 marker had been replaced by the $h p h E C 6$ marker (pFA6a-Sc_Aput1hphEC6) was generated by first excising the kanMX4 marker from the pFA6a-Sc_Aput1-kanMX4 plasmid through digestion with BglII and EcoRV. The hphEC6 cassette was amplified from the pUC57-hphECO plasmid with primers EcTEF1 5' 366 fwd2 and EcTEF1 3' 155 rev. The hphEC6 amplification product was then digested with BamHI and EcoRV and ligated to the BamHI/EcoRV-cut pFA6a-Sc_Aputl fragment.

An alternative kanMX4/hphEC6 integration plasmid (pFA6a-hphEC6-Sc_Aput1-kanMX4) was generated by amplifying the hphEC6 cassette from the pUC57-hphECO plasmid with primers EcTEF1 5' 366 fwd2 and EcTEF1 3' $155 \mathrm{rev}$, which was then digested with BamHI and EcoRV and inserted into BamHI/SmaI-cut pFA6a-Sc_sput1kanMX4 (site II in Fig. 1c).
Prior to transformation, integration plasmids were digested with SwaI to produce linearized integration constructs (Fig. 2a), which were then purified into sterile water using the QIAquick PCR purification kit (Qiagen).

\section{Yeast transformation}

The transgenic yeast strains generated in this study are listed in Table 2. The parent yeast strain for all transformations in this study was $S$. cerevisiae CBS 8340 (syn. CEN. PK 113-7D; genotype MATa MAL2-8 $8^{c}$ SUC2), which was purchased from Centraalbureau voor Schimmelcultures

Table 2 Transgenic yeast strains used in this study

\begin{tabular}{|c|c|}
\hline Strain number & $\begin{array}{l}\text { Relevant genotype (all strains } \\
\text { derived from } S \text {. cerevisiae CBS } \\
8340 \text { ) }\end{array}$ \\
\hline TLSC001 & PUT1::kanMX4-pFA6a \\
\hline TLSC002 & PUT1::kanMX4-EC1-pFA6a \\
\hline TLSC003 & PUT1::kanMX4-hphEC1-pFA6a \\
\hline TLSC004 & PUT1::kanMX4-hphEC2-pFA6a \\
\hline TLSC005 & PUT1::kanMX4-hphEC3-pFA6a \\
\hline TLSC006 & PUT1::kanMX4-hphEC4-pFA6a \\
\hline TLSC007 & PUT1::kanMX4-hphEC5-pFA6a \\
\hline TLSC008 & PUT1::kanMX4-hphEC6-pFA6a \\
\hline TLSC009 & PUT1::hphEC6-pFA6a \\
\hline TLSC010 & PUT1::kanMX4-pFA6a-hphEC6 \\
\hline
\end{tabular}

a

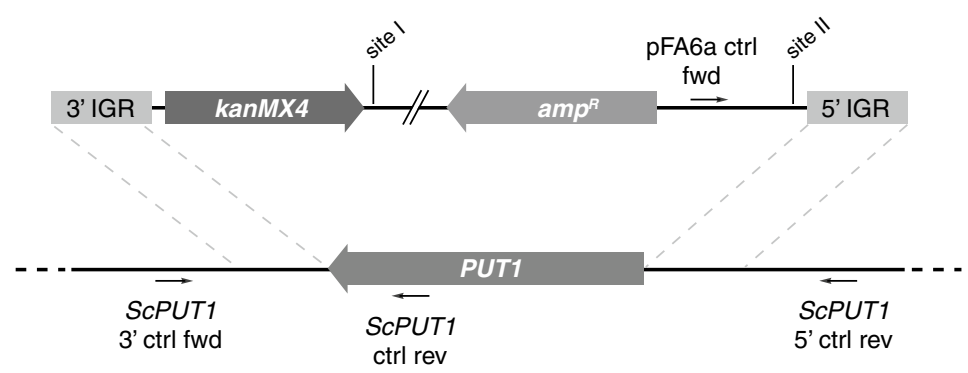

b

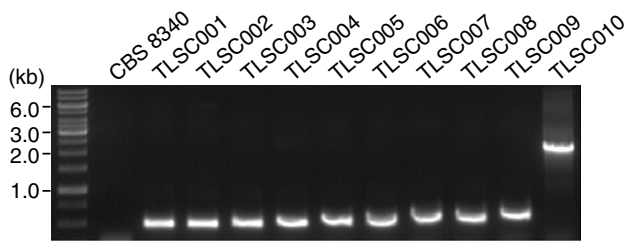

Fig. 2 Integration of plasmid constructs at the S. cerevisiae PUT1 locus. a pFA6a-Sc_Aput1-kanMX4 plasmid was linearized by digestion with SwaI to enable homologous recombination with PUT1 5' and 3' IGRs. The locations of control primers to confirm correct integration of the construct are indicated. DNA elements are not drawn to

\section{C}

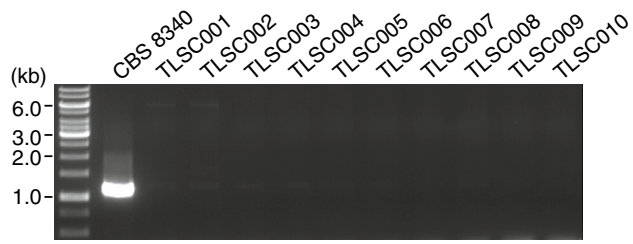

scale. b Confirmation of the correct integration of constructs as demonstrated by PCR of genomic DNA using primers pFA6a fwd and SCPUT1 5' ctrl rev. c Confirmation of the removal of the endogenous $P U T 1$ locus as demonstrated by PCR of genomic DNA using primers SCPUT1 3' fwd and SCPUT1 ctrl rev 
(Utrecht, The Netherlands). The selection agents G418 disulfate and hygromycin B were purchased from Formedium Ltd (Norfolk, UK). An aqueous stock solution of hygromycin B was prepared to a final concentration of $50 \mathrm{~g} / \mathrm{L}$, sterilized by filtration and stored at $4{ }^{\circ} \mathrm{C}$. An aqueous stock solution of G418 disulfate was prepared to a final concentration of $100 \mathrm{~g} / \mathrm{L}$, sterilized by filtration, and stored as aliquots at $-20{ }^{\circ} \mathrm{C}$.

The transformation protocol used in this study is a simplified version of the standard lithium acetate protocol. A S. cerevisiae pre-culture was diluted to a final $\mathrm{OD}_{600}$ of 0.1 in $50-\mathrm{mL}$ fresh $\mathrm{YM}$ broth $(3-\mathrm{g} / \mathrm{L}$ yeast extract, $3-\mathrm{g} / \mathrm{L}$ malt extract, 5-g/L peptone, and 10-g/L glucose) supplemented with $75-\mathrm{mg} / \mathrm{L}$ carbenicillin and incubated at $30{ }^{\circ} \mathrm{C}$ with shaking at $200 \mathrm{rpm}$ until $\mathrm{OD}_{600}$ reached 0.5-0.6. Cells were collected by centrifugation $(2500 \times g, 5 \mathrm{~min})$ and resuspended in $1.5-\mathrm{mL}$ sterile water. The washed cells were collected by centrifugation $(6900 \times g, 3 \mathrm{~min})$, the supernatant removed and the pellet resuspended in 0.4$\mathrm{mL}$ 100-mM lithium acetate. The resuspended cells were incubated at $30{ }^{\circ} \mathrm{C}$ for $15 \mathrm{~min}$ and then divided into $50-\mu \mathrm{L}$ aliquots. $10-\mu \mathrm{L}$ purified SwaI-digested integration plasmid was added to each aliquot of cells, which were then incubated at $30{ }^{\circ} \mathrm{C}$ for $15 \mathrm{~min} .0 .3 \mathrm{~mL}$ of a $100 \mathrm{mM}$ lithium acetate/40\% (w/v) PEG3350 mixture was added to each sample, and mixed and incubated for a further $15 \mathrm{~min}$ at $30{ }^{\circ} \mathrm{C}$. Samples were then incubated for $15 \mathrm{~min}$ at $42{ }^{\circ} \mathrm{C}$ followed by immediate centrifugation $(6900 \times g, 3 \mathrm{~min})$ and removal of the supernatant. $0.5 \mathrm{~mL}$ of fresh YM broth was added to each sample, which was left to stand for $5 \mathrm{~min}$ at room temperature before being fully resuspended. Each sample of $0.5-\mathrm{mL}$ resuspended cells were transferred to a fresh tube containing $2.5-\mathrm{mL}$ fresh YM broth and incubated at $30{ }^{\circ} \mathrm{C}$ for $1 \mathrm{~h}$ in a rotary shaker set to $200 \mathrm{rpm}$. Samples were then centrifuged $(6900 \times g, 3 \mathrm{~min})$ and the supernatant discarded. The cells in each sample were resuspended in 0.2-mL fresh YM broth and spread on solid YM medium ( $20 \mathrm{~g} / \mathrm{L}$ agar) containing either $400-\mathrm{mg} / \mathrm{L}$ G418 disulfate or 200-mg/L hygromycin B as specified in the "Results and discussion" section.

Correct chromosomal integration and the deletion of the PUT1 locus were confirmed by PCR analysis of purified genomic DNA from each strain (Fig. 2a). Successful integration at the $P U T 1$ locus was assayed using primers pFA6a ctrl fwd and ScPUT1 5' ctrl rev, which produce no product in the CBS 8340 parent strain, a 417-bp amplification product in strains TLSC001-TLSC009, and a 1980-bp amplification product TLSC010 due to the insertion of the hphEC6 cassette at site II (Fig. 2b). Successful deletion of the PUTI coding sequence was assayed using primers SCPUT1 3' ctrl fwd and $S c P U T 1$ ctrl rev, which produce a 1063-bp amplification product in the CBS 8340 parent strain and no product in strains TLSC001-TLSC010 (Fig. 2c).

\section{Comparative fitness assays of deletion strains}

The absolute fitness of G418 disulfate/hygromycin B-resistant strains TLSC008 and TLSC010 was compared by parallel cultivation in minimal glucose medium (MMD) broth consisting of $6.7 \mathrm{~g} / \mathrm{L}$ Difco yeast nitrogen base without amino acids (Becton, Dickinson, and Company) and $20 \mathrm{~g} / \mathrm{L}$ glucose. Both strains were pre-cultured in $3 \mathrm{~mL}$ MMD with $200 \mathrm{mg} / \mathrm{L} \mathrm{G} 418$ disulfate and $200 \mathrm{mg} / \mathrm{L}$ hygromycin B. Precultures were diluted to a final $\mathrm{OD}_{600}$ of 0.05 in separate 250-mL E-flasks containing 40-mL MMD broth with 200$\mathrm{mg} / \mathrm{L} \mathrm{G} 418$ disulfate and 200-mg/L hygromycin B. Samples were incubated at $30{ }^{\circ} \mathrm{C}$ with shaking at $200 \mathrm{rpm}$ and growth was monitored by measurement of $\mathrm{OD}_{600}$ every $24 \mathrm{~h}$ for 4 days. Each strain was assayed in triplicate.

The relative fitness of G418 disulfate-resistant strain TLSC001 and hygromycin B-resistant strain TLSC009 were assayed through co-cultivation of both strains in MMD broth without selection agent. Both strains were pre-cultured in 3-mL MMD containing either 200-mg/L G418 disulfate (for strain TLSC001) or 200-mg/L hygromycin B (for strain TLSC009). $1 \mathrm{~mL}$ of each pre-culture was harvested by centrifugation $(6900 \times g, 3 \mathrm{~min})$ and resuspended in an equal volume of fresh MMD broth without selection agent. Both strains were inoculated to a final $\mathrm{OD}_{600}$ of 0.01 each in a single 250-mL E-flask containing 40-mL MMD broth without selection agent and incubated at $30{ }^{\circ} \mathrm{C}$ with shaking at $200 \mathrm{rpm}$. Growth of each strain was monitored through enumeration by viable count on YM solid medium containing either $200 \mathrm{mg} / \mathrm{L} \mathrm{G} 418$ disulfate (for strain TLSC001) or $200 \mathrm{mg} / \mathrm{L}$ hygromycin B (for strain TLSC009). Co-cultures were sampled immediately following inoculation as well as after 24 and $48 \mathrm{~h}$ after inoculation. Co-culture experiments were performed in triplicate. Viable counts at each time point was performed in triplicate and the average expressed as colony forming units $(\mathrm{cfu}) / \mathrm{mL}$.

\section{Results and discussion}

The abundance of sequenced yeast genomes is a valuable resource for co-opting intergenic regulatory sequences for heterologous expression of transgenes in yeast. In this study, the promoter and terminator elements contained within the $5^{\prime}$ and $3^{\prime}$ IGRs of the E. cymbalariae TEFl gene (EcTEF1, systematic gene name Ecym_3450) were selected for the development of a new constitutive expression cassette in yeast. At the time writing, the yeast $E$. cymbalariae is the closest relative of $E$. gossypii with a sequenced genome (Wendland and Walther 2011). The EcTEF1 5' and 3' IGRs were, therefore, considered logical choices for the development of a new expression cassette considering the relatively 
short evolutionary distance to the well established EgTEF1based $M X$ cassette.

A fusion of the full-length $5^{\prime}$ and $3^{\prime}$ EcTEFI IGRs was synthesized de novo and inserted into the pUC57 cloning vector. The EcTEF1 expression cassette (from now on referred to as $E C O$ ) also included the first four codons of the EcTEF1 ORF corresponding to the amino acids MGKE (Fig. 1d). This differs slightly from the EgTEF1-derived $M X$ cassette, which includes the first eight codons of the EgTEF1 ORF corresponding to the amino acids MGKEKTHV. The rationale for including a shorter $\mathrm{N}$-terminal portion of the E. cymbalariae Tef1 peptide sequence was to reduce the possibility of interference with the $\mathrm{N}$-terminus of transgenes that could be critical to the function of the expressed protein. The EcTEF1 5' and 3' IGRs are both significantly longer than those of the E. gossypii ortholog. The EcTEF1 5' IGR consists of $713 \mathrm{bp}$ compared to $284 \mathrm{bp}$ for the EgTEF1 5' IGR (GenBank accession NC_005785, reverse complement of residues 58,674-58,957), while the EcTEF1 3' IGR consists of $359 \mathrm{bp}$ compared to $165 \mathrm{bp}$ in the EgTEF1 3' IGR (GenBank accession NC_005785, reverse complement of residues 57,132-57,296).

The 5' and 3' IGRs from E. cymbalariae and E. gossypii $T E F 1$ genes were aligned to assess their degree of sequence conservation. The majority of the EgTEF1 5' IGR sequence appeared to be conserved in the EcTEF1 5' IGR, while the EcTEF1 5' IGR contained two longer stretches of sequence that were not conserved in the EgTEF1 (Fig. S1a). The largest conserved sequence block (318 bp with 53\% identity and six short indels comprising a total of 57 internal gap positions) between the EcTEFI and EgTEF1 5' IGRs was located immediately upstream of the TEFI ORF in both species. The majority of EgTEF1 3' IGR appeared to be conserved in the EcTEF1 3' IGR with the majority of the conserved sequence contained within a single 156-bp sequence block (64\% identity with a single internal 8-bp indel), which was located immediately downstream of the TEFI ORF in both species.

Both TEF 1 5' IGRs were inspected for the presence of known conserved regulatory elements that are commonly found in the promoter sequences of ascomycete ribosomal protein (RP) genes (Tanay et al. 2005). Of previously characterized RP gene regulatory elements, a single putative Homolo-D site (TGTGACTG) as well as an immediately adjacent putative Rap1-binding site (CRCCCRTACAT) were found to be conserved in the $5^{\prime}$ end of both TEF 1 5' IGRs (Fig. S1a). A putative ribosomal RNA processing element (RRPE) motif (AAAAATTTT) was detected in the $E$. cymbalariae TEF 1 5' IGR, which was not conserved in the E. gossypii TEF1 5' IGR (Fig. S1a). The 6-bp core sequence AATTTT of the RRPE motif is also associated with the regulation of genes required for rapid growth in budding yeasts (Ihmels et al. 2005) and is referred to as the rapid growth element (RGE). A putative RGE motif was detected in the
E. cymbalariae TEF1 5' IGR located just upstream of the putative RRPE/RGE motif. This RGE site was not conserved in the E. gossypii TEF1 5' IGR either. Further inspection of the full-length $E$. gossypii TEF1 5' IGR sequence identified a single putative RGE motif in the reverse orientation. However, this site overlapped with a conserved region in the E. cymbalariae TEF 1 5' IGR sequence, which may indicate that it is not a de facto regulatory element.

It is possible that the 318-bp conserved sequence block just immediately upstream of the TEF 1 ORF in both species may contain novel regulatory elements important for the proper expression of the TEF1 gene. The genus Eremothecium belongs to the family Saccharomycetaceae, which includes several species with sequenced genomes such as S. cerevisiae, lactose-fermenting biotechnology yeasts of the genus Kluyveromyces, and osmotolerant spoilage yeasts of the genus Zygosaccharomyces among others. The abundance of available genomic sequences from species belonging to the Saccharomycetaceae enables the mining of genomic data for the presence of novel conserved motifs in the TEF1 gene. However, the TEF1 gene is found in two genomic contexts within the Saccharomycetaceae. In species belonging to the genera Eremothecium, Kluyveromyces, Lachancea, Torulaspora, and Zygosaccharomyces, the TEF1 gene is arranged in a tandem orientation with its neighboring upstream and downstream genes $M R L 1$ and $M U D 1$, respectively (Fig. S1b). Conversely, the TEF1 gene is present in two near-identical paralogous copies (TEF1 and TEF2) in the genera Kazachstania, Naumovozyma, Saccharomyces, Tetrapisispora, and Vanderwaltozyma. These genera constitute a monophyletic group originating from an ancient whole-genome duplication (WGD) event (Kellis et al. 2004). The WGD group TEF1 paralog has retained the ancestral MRL1-TEF1 IGR, while the TEF2 paralog has retained the ancestral TEF1-MUD1 IGR (Fig. S1c).

To scan for conserved sequence motifs in the TEF1 5' IGR among the Saccharomycetaceae, the ancestral MRL1TEF1 IGR (with the MRL1 and TEF1 genes in a tandem orientation) must first be distinguished from the more recently derived TKL2-TEF2 IGR found within the WGD group (with the TKL2 and TEF2 genes in a divergent orientation). In the latter case, the TKL2 and TEF2 genes share a common bidirectional promoter, which could introduce unwanted noise into the motif elucidation algorithm. Consequently, all TKL2-TEF2 IGR sequences from WGD species of the Saccharomycetaceae were excluded from the further analysis in the current study. MRL1-TEF1 IGR sequences from 24 species belonging to the Saccharomycetaceae (Eremothecium coryli, E. cymbalariae, E. gossypii, Kazachstania africana, Kazachstania naganishii, Kluyveromyces aestuarii, Kluyveromyces lactis, Kluyveromyces marxianus, Kluyveromyces wickerhamii, Lachancea kluyveri, Lachancea lanzarotensis, Lachancea quebecensis, Lachancea 
thermotolerans, Lachancea waltii, Naumovozyma castellii, Naumovozyma dairenensis, S. cerevisiae, Saccharomyces eubayanus, Saccharomyces paradoxus, Tetrapisispora blattae, Tetrapisispora phaffii, Torulaspora delbrueckii, Zygosaccharomyces bailii, and Zygosaccharomyces rouxii) were assayed for the overrepresentation of sequence motifs using MEME (http://meme-suite.org/tools/meme; Bailey et al. 2009). The only significant hits were the previously identified RRPE motif, which was detected in 19 out the 24 input sequences, and the shorter RGE motif, which was found in all 24 sequences. Putative Homolo-D and Rap1 sites were only found in a smaller sub-set of the sequences. No novel sites above the significance threshold $\left(E=10^{-3}\right)$ were identified.

The greater lengths of the $5^{\prime}$ and 3' IGRs of the EcTEFI gene as compared to those of the EgTEF1 gene may be problematic for some applications: for example, decreased PCR amplification efficiency of longer linear DNA constructs. Such problems could be circumvented by trimming one or both IGRs of the EcTEF1 gene as long as sufficient functionality could be retained. To test the potential of using trimmed variants of the $E C O$ cassette, the plasmid-encoded hygromycin B phosphotransferase gene $h p h$ from the bacterium Escherichia coli was selected for expression. Hygromycin B is an aminoglycoside antibiotic produced by the actinobacterium Streptomyces hygroscopicus (Mann and Bromer 1958), which inhibits protein synthesis in both bacteria and eukaryotes. Expression of the $h p h$ gene has previously been shown to confer resistance to hygromycin B in both bacteria and yeast (Gritz and Davies 1983; Kaster et al. 1984).

The $h p h$ coding sequence was inserted into the ECO cassette to generate the $h p h E C O$ cassette. A spectrum of $h p h E C$ variants ( $h p h E C l$ through $h p h E C 6$ ) with progressively trimmed $5^{\prime}$ and $3^{\prime}$ IGR segments was subsequently generated by PCR amplification using different primer combinations (Fig. 1e, Fig. S1a). Each $h p h E C$ variant was then inserted into the integration construct pFA6a-Sc_Aput1kanMX4 (Fig. 1c), so that the hphEC cassette was located immediately downstream of the kanMX4 selection marker in a tandem orientation. The $S$. cerevisiae proline oxidaseencoding PUT1 locus was selected as the integration site for this study as the gene is only essential for cell viability when L-proline is the only available source of nitrogen (Wang and Brandriss 1986), which allows for convenient phenotypical confirmation of transformants. A control construct containing an empty $E C l$ cassette (pFA6a-Sc_Aputl-kanMX4-ECl) was included to confirm that the EcTEFI IGRs did not confer cryptic resistance to hygromycin B.

The integration constructs were used to transform $S$. cerevisiae with G418 disulfate used for selection of positive transformants. Plate growth assays of confirmed transformants containing any of the six $h p h E C$ variants (strains TLSC003-TLSC008) were able to grow on solid YM medium containing up to $400 \mathrm{mg} / \mathrm{L}$ hygromycin $\mathrm{B}$, which was the highest concentration tested (Fig. 3). No significant growth was observed on hygromycin B-containing medium $(100-400 \mathrm{mg} / \mathrm{L})$ for strains TLSC001 and TLSC002, which had been transformed with the control constructs pFA6aSc_Aput1-kanMX4 and pFA6a-Sc_Aput1-kanMX4-EC1, respectively.

The previously observed putative Homolo-D and Rap1 sites in the EcTEF1 5' IGR (Fig. S1a) were shown to be dispensable for sufficient expression of the $h p h$ gene in $S$. cerevisiae to enable growth at hygromycin B concentrations up to $400 \mathrm{mg} / \mathrm{L}$ as demonstrated by $h p h E C$ variants $h p h E C 3$ through $h p h E C 6$. This does not exclude the possibility that these two motifs do play a role in the proper regulation of the endogenous TEFI gene in E. cymbalariae. Another possibility is that the MRL1 gene, which is located immediately upstream of TEF1 in the E. cymbalariae genome (Fig. S1b), is the regulatory target of these sites. However, the $M R L 1$ gene encodes a putative membrane protein similar to mammalian mannose-6-phosphate receptors with a possible function as a sorting receptor in the delivery of vacuolar hydrolases (Whyte and Munro 2001) but does not appear to have any obvious functional connection to protein synthesis. In addition, the E. cymbalariae MRL1 ortholog (systematic gene name Ecym_3451) is annotated as nonfunctional with a reported frame-shift mutation. The tandem orientation of the MRL1 and TEF 1 genes would also suggest that the conserved Homolo-D and Rap1-binding sites in the

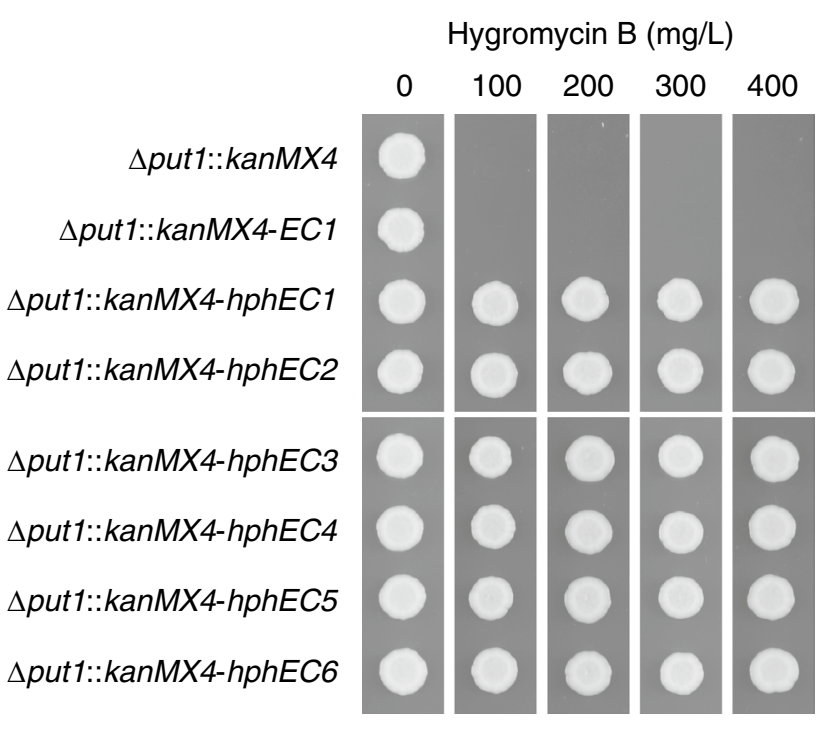

Fig. 3 Hygromycin B tolerance of $h p h E C$ variants. Strains were precultured overnight in $3 \mathrm{~mL}$ YM broth with $200 \mathrm{mg} / \mathrm{L} \mathrm{G} 418$ disulfate and then diluted to $\mathrm{OD}_{600} 0.1$ in fresh YM broth without any selection agent. $2 \mu \mathrm{L}$ cell suspension of each strain was spotted on solid YM medium with the indicated concentration of hygromycin B. Plates were incubated for 2 days at $30^{\circ} \mathrm{C}$ and then photographed

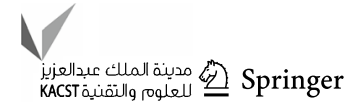


MRL1-TEF1 IGR, if functional, are not involved in regulating the expression of the MRL1 gene.

All remaining experiments of the $h p h E C$ cassette within the current study utilized the shortest variant, hphEC6. The next question to be addressed was whether the adjacent kanMX4 and hphEC6 cassettes in the pFA6a-Sc_Aput1kanMX4-hphEC6 construct interacted in any positive manner or negative manner. For example, would the hphEC6 selection marker retain its activity in the absence of the kanMX4 cassette? Likewise, would the close proximity of the kanMX4 and hphEC6 cassettes in the pFA6a-Sc_Aput 1 kanMX4-hphEC6 construct cause any detectable interference between the two selection markers?

Two additional hphEC6-containing constructs were, therefore, generated to investigate any potential interaction between the hphEC6 and kanMX4 cassettes in the
pFA6a-Sc_Aput1-kanMX4-hphEC6 construct. The first construct replaced the kanMX4 cassette in the pFA6a-Sc_Aput1kanMX4 plasmid with the $h p h E C 6$ cassette to produce the plasmid pFA6a-Sc_Aput1-hphEC6 (Fig. 4a). This construct would enable the evaluation of the hphEC6 marker both as a selection marker for transformation on hygromycin B-containing medium and also allow functional study of the hphEC6 cassette in the absence of the kanMX4 cassette. The transformation of $S$. cerevisiae CBS 8340 was repeated with the pFA6a-Sc_Aput1-hphEC6 construct with $200 \mathrm{mg} / \mathrm{L}$ hygromycin $B$ as the selection agent rather than $400 \mathrm{mg} / \mathrm{L}$ G418 disulfate. Ten random colonies were picked from the selective media and screened for the presence of the pFA6aSc_Aputl-hphEC6 construct at the PUTl locus by PCR as before (Fig. 2b, c). All ten transformants were found to have successfully integrated the pFA6a-Sc_Aput1-hphEC6
Fig. 4 Assaying interactions and relative fitness between kanMX4 and hphEC6 selection markers. a Outline of linearized constructs used in interaction assays. Plasmid elements are not drawn to scale. $\mathbf{b}$ Tolerance of yeast strains to combinations of G418 sulfate and hygromycin B. Strains were pre-cultured overnight in $3 \mathrm{~mL}$ YM broth with either $200 \mathrm{mg} / \mathrm{L} \mathrm{G} 418$ disulfate (TLSC001), $200 \mathrm{mg} / \mathrm{L}$ hygromycin B (TLSC009), or $200 \mathrm{mg} / \mathrm{L}$ of both selection agents (TLSC008 and TLSC010). Pre-cultures were diluted to $\mathrm{OD}_{600} 0.1$ in fresh YM broth without any selection agent. $2 \mu \mathrm{L}$ cell suspension of each strain was spotted on solid YM medium with the indicated concentration of G418 disulfate and hygromycin B. Plates were incubated for 2 days at $30{ }^{\circ} \mathrm{C}$ and then photographed. c Comparative growth dynamics in MMD broth containing both G418 disulfate and hygromycin B. Error bars indicate one standard deviation. d Fitness equivalence of co-cultivated S. cerevisiae $\Delta$ put 1 strains carrying either the kanMX4 or $h p h E C 6$ marker under nonselective conditions. Error bars indicate one standard deviation

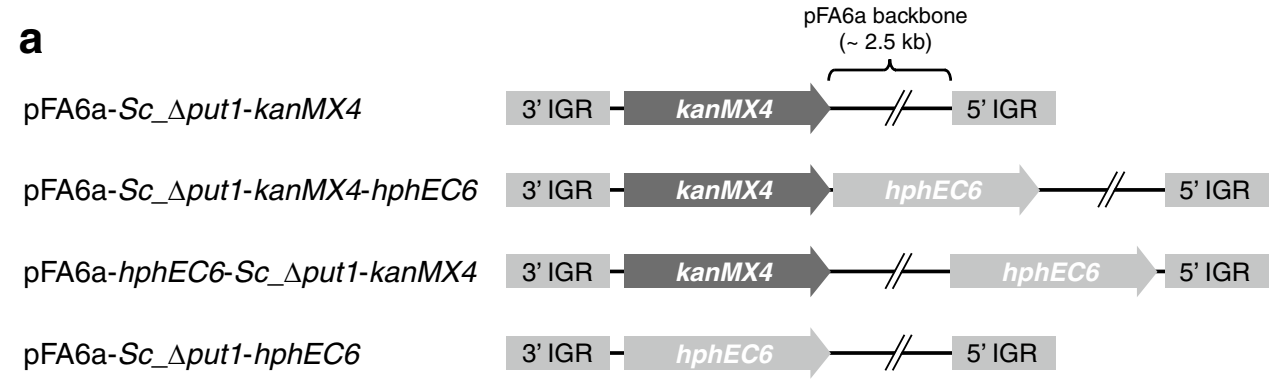

b

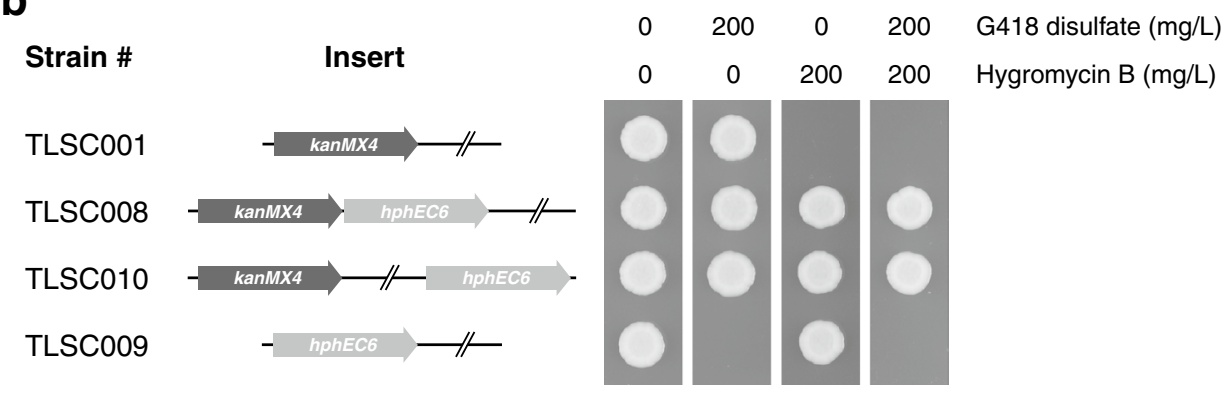

C

d
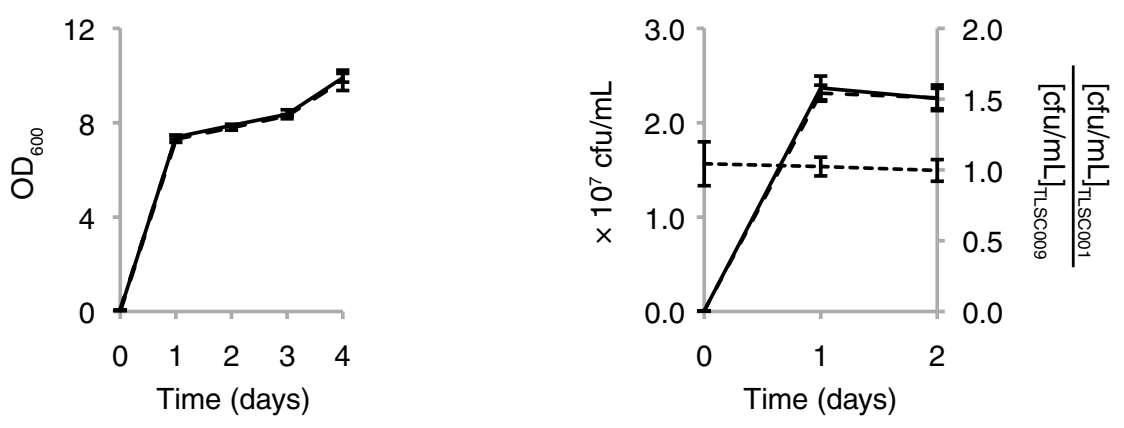

- TLSC008
- TLSC010

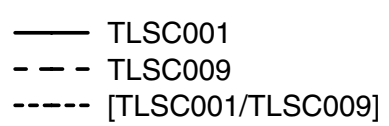


construct and replaced the endogenous PUT1 locus, which demonstrated the utility of the hphEC6 as a hygromycin B-dependent selection marker. One of the transformants was selected for subsequent assays and designated strain TLSC009.

A second construct was designed where the hphEC6 cassette was inserted at site II in pFA6a-Sc_Aput1-kanMX4 (Fig. 1c) to make plasmid pFA6a-hphEC6-Sc_sput1kanMX4 (Fig. 4a). Once the linearized pFA6a-hphEC6Sc_Aput1-kanMX4 construct had been integrated into the S. cerevisiae genome to make strain TLSC010, the kanMX4 and $h p h E C 6$ cassettes would be separated by the entire length of the pFA6a plasmid backbone (2479 bp). The tolerance of strains TLSC009 and TLSC010 to G418 disulfate and hygromycin B were tested using a plate growth assay and compared to strains TLSC001 and TLSC008 (Fig. 4b). As expected, strain TLSC009 was sensitive to G418 at $200 \mathrm{mg} / \mathrm{L}$, while strain TLSC010 was resistant to both G418 disulfate and hygromycin B at $200 \mathrm{mg} / \mathrm{L}$.

Although strains TLSC008 and TLSC010 displayed similar growth on solid medium containing both G418 disulfate and hygromycin $\mathrm{B}$, the agar growth assay was only considered semi-quantitative. A growth assay in liquid medium was, therefore, performed to investigate whether the proximity between the kanMX4 and hphEC6 cassettes in strains TLSC008 and TLSC010 had any subtle effect of strain fitness when both resistance markers are under selection. Both strains were cultivated in parallel using a chemically defined liquid minimal medium containing both G418 disulfate and hygromycin $\mathrm{B}$. The growth dynamics of both strains were monitored by optical density measurements every $24 \mathrm{~h}$ and shown to be both highly reproducible and indistinguishable from each other (Fig. 4c). It was, therefore, concluded that the $h p h E C 6$ cassette could be placed immediately adjacent to the $k a n M X 4$ cassette in a tandem orientation without any discernable effects. However, the kanMX4 and hphEC6 cassettes were not tested in a convergent orientation in this study and there is a possibility that the selection markers could affect the activity of each other in such an orientation.

The final question to be resolved in the present study was whether the hphEC6 cassette was functionally equivalent to the kanMX4 cassette in terms of strain fitness under nonselective conditions where neither selection agent is present. Relative fitness was assayed through co-cultivation of strains TLSC001 and TLSC009, which are isogenic with exception of carrying either the kanMX4 or hphEC6 cassette, respectively (Fig. 4a). Both strains were inoculated in chemically defined liquid minimal medium lacking any selection agent with an initial $\mathrm{OD}_{600}$ of 0.01 corresponding to $65,000-70,000 \mathrm{cfu} / \mathrm{mL}$ of each strain. Growth of both strains was monitored separately through enumeration by viable count on solid-rich medium containing either G418 disulfate or hygromycin B, respectively. Both strains had reached stationary phase within $24 \mathrm{~h}$ and no significant deviation from the initial TLSC001/TLSC009 inoculation ratio $(1.04 \pm 0.16)$ was observed at either 24 or $48 \mathrm{~h}$ after inoculation (Fig. 4d). It could not be excluded that significant differences in fitness between the selection markers would be observed after prolonged incubation in stationary phase. Likewise, such differences in fitness may become apparent only after repeated cycles of batch co-cultivations. However, for standard genetic applications such as heterologous expression of dominant selection markers to enable gene replacement, the kanMX4 and hphEC6 selection markers appear to be essentially equal in fitness.

\section{Conclusion}

The present study has demonstrated the suitability of the $E c T E F 1$-derived expression cassette $E C O$ for the design of dominant selection markers in yeast engineering. The EcTEF 1 5' and ' ' IGRs could be trimmed down to at least 366 and $155 \mathrm{bp}$, respectively, while retaining resistance to at least $400 \mathrm{mg} / \mathrm{L}$ hygromycin B when expressing the $h p h$ hygromycin phosphotransferase gene (Fig. 3). Detailed expression dynamics of different variants of the $E C$ cassette with regard to growth phase and nutritional status were not investigated in this study. Such information may be of importance when employing the cassette for the expression of transgenes for other purposes than dominant selection markers and would need to be determined on a case-by-case basis both in terms of transgene and strain background. Concerning the latter aspect, the differences in RP gene regulatory elements in different ascomycete fungi (Tanay et al. 2005) need to be considered as they may affect expression dynamics in a species-dependent manner.

\section{Compliance with ethical standards}

Ethical approval This article does not contain any studies with human participants or animals performed by the author.

Conflict of interest The author declares no conflict of interest.

Open Access This article is distributed under the terms of the Creative Commons Attribution 4.0 International License (http://creativeco mmons.org/licenses/by/4.0/), which permits unrestricted use, distribution, and reproduction in any medium, provided you give appropriate credit to the original author(s) and the source, provide a link to the Creative Commons license, and indicate if changes were made. 


\section{References}

Ahn J, Hong J, Lee H, Park M, Lee E, Kim C, Choi E, Jung J, Lee H (2007) Translation elongation factor 1-alpha gene from Pichia pastoris: molecular cloning, sequence, and use of its promoter. Appl Microbiol Biotechnol 74:601-608

Bähler J, Wu JQ, Longtine MS, Shah NG, McKenzie A, Steever AB, Wach A, Philippsen P, Pringle JR (1998) Heterologous modules for efficient and versatile PCR-based gene targeting in Schizosaccharomyces pombe. Yeast 14:943-951

Bailey TL, Boden M, Buske FA, Frith M, Grant CE, Clementi L, Ren JY, Li WW, Noble WS (2009) MEME SUITE: tools for motif discovery and searching. Nucleic Acids Res 37:W202-W208

Beimforde C, Feldberg K, Nylinder S, Rikkinen J, Tuovila H, Dörfelt H, Gube M, Jackson DJ, Reitner J, Seyfullah LJ, Schmidt AR (2014) Estimating the Phanerozoic history of the Ascomycota lineages: combining fossil and molecular data. Mol Phylogenet Evol 78:386-398

Davidson JF, Schiestl RH (2000) Mis-targeting of multiple gene disruption constructs containing hisG. Curr Genet 38:188-190

Ghaemmaghami S, Huh WK, Bower K, Howson RW, Belle A, Dephoure N, O'Shea EK, Weissman JS (2003) Global analysis of protein expression in yeast. Nature 425:737-741

Gritz L, Davies J (1983) Plasmid-encoded hygromycin B resistance: the sequence of hygromycin B phosphotransferase gene and its expression in Escherichia coli and Saccharomyces cerevisiae. Gene 25:179-188

Güldener U, Heck S, Fielder T, Beinhauer J, Hegemann JH (1996) A new efficient gene disruption cassette for repeated use in budding yeast. Nucleic Acids Res 24:2519-2524

Ihmels J, Bergmann S, Gerami-Nejad M, Yanai I, McClellan M, Berman J, Barkai N (2005) Rewiring of the yeast transcriptional network through the evolution of motif usage. Science 309:938-940

Janke C, Magiera MM, Rathfelder N, Taxis C, Reber S, Maekawa H, Moreno-Borchart A, Doenges G, Schwob E, Schiebel E, Knop M (2004) A versatile toolbox for PCR-based tagging of yeast genes: new fluorescent proteins, more markers and promoter substitution cassettes. Yeast 21:947-962
Kaster KR, Burgett SG, Ingolia TD (1984) Hygromycin B resistance as dominant selectable marker in yeast. Curr Genet 8:353-358

Kellis M, Birren BW, Lander ES (2004) Proof and evolutionary analysis of ancient genome duplication in the yeast Saccharomyces cerevisiae. Nature 428:617-624

Mann RL, Bromer WW (1958) The isolation of a second antibiotic from Streptomyces hygroscopicus. J Am Chem Soc 80:2714-2716

Müller S, Thomas S, Peter KH, Henrik D (1998) Comparison of expression systems in the yeast Saccharomyces cerevisiae, Hansenula polymorpha, Kluyveromyces lactis, Schizosaccharomyces pombe and Yarrowia lipolytica. Cloning of two novel promoters from Yarrowia lipolytica. Yeast 14:1267-1283

Solis-Escalante D, van den Broek M, Kuijpers NG, Pronk JT, Boles E, Daran JM, Daran-Lapujade P (2015) The genome sequence of the popular hexose-transport-deficient Saccharomyces cerevisiae strain EBYVW4000 reveals LoxP/Cre-induced translocations and gene loss. FEMS Yeast Res 15 pii:fou 004

Steiner S, Philippsen P (1994) Sequence and promoter analysis of the highly expressed $T E F$ gene of the filamentous fungus Ashbya gossypii. Mol Gen Genet 242:263-271

Tanay A, Regev A, Shamir R (2005) Conservation and evolvability in regulatory networks: the evolution of ribosomal regulation in yeast. Proc Natl Acad Sci USA 102:7203-7208

Wach A, Brachat A, Pohlmann R, Philippsen P (1994) New heterologous modules for classical or PCR-based gene disruptions in Saccharomyces cerevisiae. Yeast 10:1793-1808

Wang SS, Brandriss MC (1986) Proline utilization in Saccharomyces cerevisiae: analysis of the cloned PUT1 gene. Mol Cell Biol 6:2638-2645

Wendland J, Walther A (2011) Genome evolution in the eremothecium clade of the Saccharomyces complex revealed by comparative genomics. G3 1:539-548

Whyte JR, Munro S (2001) A yeast homolog of the mammalian mannose 6-phosphate receptors contributes to the sorting of vacuolar hydrolases. Curr Biol 11:1074-1078 